promotes tumor growth. Cancer Res. 65:2277-2286. 12. Covello, K.L., et al. 2006. HIF-2alpha regulates Oct-4: effects of hypoxia on stem cell function, embryonic development, and tumor growth. Genes Dev. 20:557-570

13. Kondo, K., Kico, J., Nakamura, E., Lechpammer, M., and Kaelin, W.G.J. 2002. Inhibition of HIF is necessary for tumor suppression by the von Hippel-Lindau protein. Cancer Cell. 1:237-246.

14. Raval, R.R., et al. 2005. Contrasting properties of hypoxia-inducible factor 1 (HIF-1) and HIF-2 in von Hippel-Lindau-associated renal cell carcinoma. Mol. Cell. Biol. 25:5675-5686.
15. Park, S.-K., et al. 2003. Hypoxia-induced gene expression occurs solely through the action of hypoxiainducible factor 1a (HIF-1a): role of cytoplasmic trapping of HIF-2a. Mol. Cell. Biol. 23:4959-4971.

16. Hu, C.J., et al. 2006. Differential regulation of the transcriptional activities of hypoxia-inducible factor 1 alpha (HIF- $1 \alpha$ ) and HIF- $2 \alpha$ in stem cells. Mol. Cell. Biol. 26:3514-3526.

17. Yu, A.Y., et al. 1999. Impaired physiological responses to chronic hypoxia in mice partially deficient for hypoxia-inducible factor $1 \alpha$. J. Clin. Invest. 103:691-696

18. Scortegagna, M., et al. 2005. HIF-2alpha regulates murine hematopoietic development in an erythropoietin-dependent manner. Blood. 105:3133-3140.

19. Warnecke, C., et al. 2004. Differentiating the functional role of hypoxia-inducible factor (HIF)-1alpha and HIF-2alpha (EPAS-1) by the use of RNA interference: erythropoietin is a HIF-2alpha target gene in HepB and Kelly cells. FASEB J. 18:1462-1464.

20. Maxwell, P.H., et al. 1993. Identification of the renal erythropoietin-producing cells using transgenic mice. Kidney Int. 44:1149-1162.

21. Da Silva, J.L., et al. 1990. Tumour cells are the site of erythropoietin synthesis in human renal cancers associated with polycythemia. Blood. 75:577-582.

\title{
HOX deregulation in acute myeloid leukemia
}

\author{
Kim L. Rice and Jonathan D. Licht \\ Division of Hematology/Oncology, Department of Medicine, Robert H. Lurie Comprehensive Cancer Center, \\ Feinberg School of Medicine, Northwestern University, Chicago, Illinois, USA.
}

\begin{abstract}
The deregulation of bomeobox (HOX) genes in acute myeloid leukemia (AML) and the potential for these master regulators to perturb normal hematopoiesis is well established. To date, overexpression of $\mathrm{HOX}$ genes in AML has been attributed to specific chromosomal aberrations and abnormalities involving mixed-lineage leukemia (MLL), an upstream regulator of $H O X$ genes. The finding reported in this issue of the JCI by Scholl et al. that caudal-type homeobox transcription factor 2 (CDX2), which is capable of affecting $\mathrm{HOX}$ gene expression during embryogenesis, is overexpressed in $90 \%$ of patients with AML and induces a transplantable AML in murine models provides an alternative mechanism for HOX-induced leukemogenesis and yields important insights into the hierarchy of $H O X$ gene regulation in AML (see the related article beginning on page 1037).
\end{abstract}

Acute myeloid leukemia (AML) is a heterogeneous disease in which hematopoietic progenitor cells acquire genetic lesions that lead to a block in differentiation, increased self-renewal, and unregulated proliferation. The emergence of leukemic blasts appears to require at least two major genetic "hits," involving perturbations in growth factor signaling pathways and hematopoietic differentiation programs (1).

Among the receptor tyrosine kinases (RTKs), fms-like tyrosine kinase 3 (FLT3), which plays important roles in hematopoietic progenitor cell survival and

Nonstandard abbreviations used: AML, acute myeloid leukemia; ATRA, all-trans retinoic acid; CDX2, caudal-type homeobox transcription factor 2; ETV6, ets variant gene 6; FLT3, fms-like tyrosine kinase 3; HOX, homeobox; MEIS1, myeloid ecotropic viral integration site 1; MLL, mixed-lineage leukemia; PML, promyelocytic leukemia; RAR $\alpha$, retinoic acid receptor $\alpha$; RTK, receptor tyrosine kinase; RUNX1, runt-related transcription factor 1 .

Conflict of interest: The authors have declared that no conflict of interest exists.

Citation for this article: J. Clin. Invest. 117:865-868 (2007). doi:10.1172/JCI31861. proliferation, is overexpressed in a significant proportion of AMLs, and mutations resulting in the constitutive activation of FLT3 occur in approximately 33\% of patients (2). Mutations leading to the constitutive activation of a related RTK, c-KIT, and of signaling intermediates such as RAS, are also frequently described in AML (3). The dysregulation of associated signaling pathways (e.g., Ras/MAPK, $\mathrm{PI} 3 \mathrm{~K} / \mathrm{AKT}$, and JAK/STAT) is thought to result in growth factor-independent proliferation and clonal expansion of hematopoietic progenitors.

The second hit targets transcription factors capable of disrupting hematopoietic cell differentiation. This may occur following the dysregulation of specific gene regulators as a result of gene amplification (e.g., $v$-myc myelocytomatosis viral oncogene homolog $[M Y C]$, mixed-lineage lenkemia $[M L L]$, genes at the chromosome 11q23 locus) (4); point mutations in transcriptional regulators (e.g., CCAAT/enhancer-binding protein $[C / E B P]$, runt-related transcription factor 1 [RUNX1]); and chromosomal transloca- tions resulting in the fusion of promyelocytic lenkemia $(P M L)$ and the retinoic acid receptor $\alpha(R A R a)$ to yield the PML-RAR $\alpha$ chimeric protein or fusion of RUNX1 and runt-related transcription factor 1, translocated to 1 (RUNX1T1), which gives rise to the RUNX1-RUNX1T1 fusion protein (1). It is becoming increasingly clear that one set of genes commonly affected by these chimerical and mutated transcriptional regulators are the homeobox (HOX) genes. Accordingly, overexpression of homeobox master transcription factors, which fulfill critical roles in embryonic development, organogenesis, and normal hematopoietic differentiation, is a common feature of AML (5).

\section{HOX genes: from hematopoiesis to leukemia}

In mammals, HOX genes are located in two main clusters, the primordial cluster and the ParaHox cluster, which are thought to originate from the duplication of a hypothetical ProtoHox cluster of four genes early in evolution (6). The primordial HOX cluster consists of 13 paralogous groups of genes that exist as distinct, unlinked complexes on human chromosomes $7 \mathrm{p} 15$ (HOXA), 17q21 (HOXB), 12q13 (HOXC), and $2 \mathrm{q} 31(H O X D)$, and the cluster is organized such that during embryonic development, the order of expression along the anterior-posterior embryonic axis ( $3^{\prime}$ to $5^{\prime}$ ) is colinear with the alignment of genes on the chromosome (Figure 1). During hematopoiesis, HOX genes are expressed in lineage- and stage-specific combinations; however, cell commitment to myeloid or erythroid lineages is accom- 


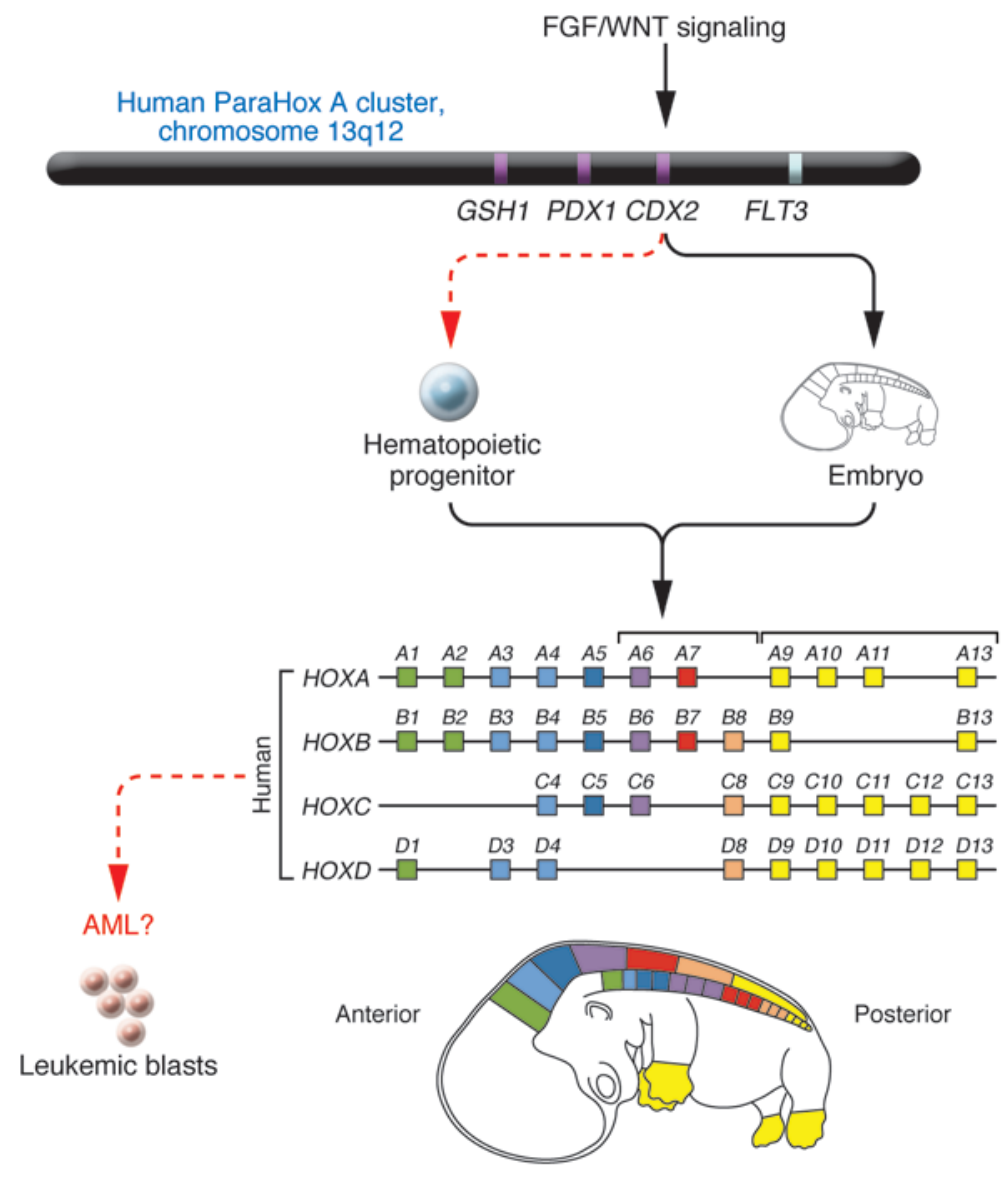

panied by global downregulation of $H O X$ gene expression (7).

HOX genes may be dysregulated in AML by several different mechanisms. First, specific $H O X$ genes can be disrupted via chromosomal translocation. Specifically, HOXA9 and HOXD13 are dysregulated through the $\mathrm{t}(7 ; 11)$ and $\mathrm{t}(2 ; 11)$ translocations, respectively (8), in both cases creating fusion proteins between the HOX protein and the nucleoporin $98 \mathrm{kDa}$ (NUP98) nuclear protein. Overexpression of HOXA6, HOXA7, HOXA9, and the HOX cofactor myeloid ecotropic viral integration site 1 (MEIS1) has also been correlated with chromosome 11q23 abnormalities involving the MLL protein, which regulates the expression of HOX genes (9). A less frequently observed translocation in $\mathrm{AML}$, $\mathrm{t}(8 ; 16)(\mathrm{p} 11 ; \mathrm{p} 13)$, which results in the overexpression of the MYST3-CREBBP fusion protein, is also associated with overexpression of HOXA9, HOXB9, HOXA10, and MEIS1 (10). In addition, the expression of HOXC4 was shown to be upregulated in the NB4 PML-RAR $\alpha$ cell line following all-trans retinoic acid-induced (ATRA- induced) differentiation as well as in bone marrow from acute PML patients during ATRA treatment, strengthening the theory that dysregulated HOX expression is a signature feature of AML (11).

\section{CDX2 overexpression: a unifying theme in AML?}

In keeping with the theme of dysregulated $H O X$ gene expression in AML, in this issue of the JCI Scholl et al. (12) demonstrate that caudal-type bomeobox transcription factor 2 (CDX2), a homeobox gene involved in anteroposterior axis definition and intestinal epithelial cell differentiation (13), but strikingly absent in hematopoietic progenitors, is overexpressed in $90 \%$ of patients with AML. The leukemogenic potential of $C D X 2$ overexpression was first described by Chase et al. (14) following the identification of a novel chromosomal rearrangement, $\mathrm{t}(12 ; 13)(\mathrm{p} 13 ; \mathrm{q} 12)$, in a patient with AML, which yielded the ets variant gene 6-CDX2 (ETV6-CDX2) fusion protein in addition to full-length CDX2. Significant$1 y$, transduction of murine hematopoietic progenitors with $\mathrm{CDX} 2$, but not the

\section{Figure 1}

A model for CDX2-mediated leukemogenesis. CDX2 is expressed in the posterior primitive streak during embryogenesis, where it directs anteroposterior axial development and elongation by regulating $H O X$ gene expression. A study in this issue of the $\mathrm{JCl}$ (12) reports that monoallelic expression of CDX2 is observed in $90 \%$ of patients with AML and may perturb hematopoiesis by affecting $H O X$ gene expression (red dashed arrows indicate ectopic expression; black arrows indicate normal expression in embryonic development). GSH1, GS homeobox 1; $P D X 1$, pancreatic and duodenal homeobox 1 . Figure modified with permission from Molecular Genetics and Metabolism (21).
ETV6-CDX2 fusion protein, resulted in a transplantable and fatal AML. Although ETV6-CDX2 induced myeloproliferation, it did not induce leukemia or accelerate CDX2-mediated leukemogenesis (15), suggesting that the primary oncogenic affect of this translocation is the ectopic expression of CDX2 in the hematopoietic compartment (Figure 1).

CDX2 belongs to the ParaHox A cluster, which consists of a three-gene complex including GS bomeobox 1 (GSH1), pancreatic and duodenal homeobox 1 (PDX1), and CDX2 that exists on chromosome 13q12 (6) (Figure 1). On the basis of sequence similarity, $C D X$ is more related to the posterior group of $H O X$ genes, which includes paralogous groups 9-13, and during embryonic development is expressed in the posterior primitive streak to regulate $H O X$ genes and in posterior signaling pathways to direct anteroposterior axis development and elongation. Given the role of CDX2 as a regulator of HOX genes in development, it is therefore not surprising that ectopic expression of CDX2 in hematopoietic progenitors perturbs blood cell differentia- 
tion programs strictly regulated by $H O X$ genes. The finding by Scholl et al. (12) that overexpression of $C d x 2$ in primary murine hematopoietic progenitors resulted in transplantable AML in vivo, coinciding with a three-fold upregulation in Hoxb6 expression, is particularly intriguing given that overexpression of HOXB6 has been documented in approximately $40 \%$ of human AMLs that do not have chromosomal translocations (16). Identifying whether $\mathrm{Cd} \mathrm{d} 2$ directly regulates Hoxb6 and providing evidence of the specific involvement of Hoxb6 in Cdx2-mediated leukemogenesis would strengthen the hypothesis that dysregulated $H O X$ gene expression by CDX2 is a major pathway contributing to leukemogenesis.

The dysregulation of HOX genes by members of the caudal HOX family may prove to be a unifying theme in AML, particularly in light of a recent study by Bansal et al. (17) that revealed dysregulated $C D X 4$ expression in $25 \%$ of AMLs. However, whereas CDX2 induced rapid AML in bone marrow transplant recipients, CDX4 induced a partially penetrant, long-latency AML, suggesting that these transcription factors regulate distinct sets of genes, including potentially different subsets of $H O X$ genes. In addition to dysregulating HOX genes, CDX2 overexpression may also affect signaling pathways. Cdx1/2 mutant embryos demonstrate posterior body truncations that phenocopy lossof-function Wnt3a and Fgfr1 mutants (18), suggesting an overlap among $\mathrm{Cdx}$, Wnt, and Fgf pathways in axial patterning. This implies that some CDX2 target genes might include components of signaling pathways or that CDX2 itself may be a target of cellsignaling regulators, a point highlighted by Scholl et al. (12), given that related family members $C D X 1$ and $C D X 4$ are direct targets of the Wnt pathway commonly activated in AML. Indeed, Scholl et al. showed that knockdown of CDX2 slowed cellular proliferation specifically in CDX2-expressing AML cell lines. Taken together, these data suggest that gain-of-function mutations of CDX2 might augment cell signaling in addition to disrupting differentiation programs. This may explain the rapid onset of AML in mice transplanted with CDX2-expressing hematopoietic progenitors.

\section{Unraveling the mechanism}

The molecular basis for the overexpression of CDX2 remains elusive, and the absence of chromosomal rearrangements or mutations in the coding sequence or proximal promoter of $C D X 2$ suggest that other undefined regulatory regions driving CDX2 expression may be a target for mutation. Indeed, aberrant expression of CDX2 in the absence of genetic abnormalities on chromosome 13 was previously documented in a case of chronic myeloid leukemia blast crisis (14). Intriguingly, microarray analysis of $50 \mathrm{AML}$ patient specimens revealed that the highest levels of $H O X$ expression are associated with a subset of patients with intermediate cytogenetics and elevated levels of FLT3 mRNA and FLT3 mutations. In the absence of mutations or amplifications, overexpression of FLT3 was correlated with aberrant expression of multiple $H O X$ genes, suggesting that deregulation of HOX genes may be due to aberrant growth factor signaling (19). Given the close proximity of the FLT3 gene to the CDX2 gene and the fact that both proteins are overexpressed in a high percentage of AMLs, it is tempting to speculate that cis-acting activating mutations in promoter or enhancer elements in the vicinity of these genes may contribute to the leukemic initiation and progression and may account for the link among FLT3, CDX2, and HOX gene overexpression in the absence of detectable cytogenetics (20). Detailed analyses of the regulatory sequences flanking CDX2 and FLT3 could provide insights into the role of these oncogenes in AML. It would also be important to correlate the level of CDX2 overexpression in AML with HOX gene expression.

Identification of a HOX gene pathway disrupted in a substantial proportion of patients with AML (12) is a major clue to disease pathogenesis; however, more direct evidence of a link between CDX2 overexpression and the dysregulation of specific HOX genes is needed. For example, demonstration by chromatin precipitation that CDX2 occupies the promoters of genes such as HOXB8 and HOXB6 in CDX2-positive AML cell specimens would reinforce the importance of CDX2 as an aberrant regulator of $H O X$ genes. In addition, identifying the mechanism by which CDX2 is overexpressed is crucial for unraveling the hierarchy of genetic pathways required for leukemia initiation and progression. While specific targeting of transcriptional regulators remains elusive, better understanding of the circuitry of the HOX genes in AML could present a new set of genetic targets, with potential therapeutic benefit for multiple AML subtypes.
Address correspondence to: Jonathan D. Licht, Division of Hematology/Oncology, Department of Medicine, Robert H. Lurie Comprehensive Cancer Center, Feinberg School of Medicine, Northwestern University, 303 E. Superior Street, Lurie 5-123, Chicago, Illinois 60611, USA. Phone: (312) 503-0985; Fax: (312) 503-0189; E-mail: j-licht@northwestern.edu.

1. Licht, J.D., and Sternberg, D.W. 2005. The molecular pathology of acute myeloid leukemia. Hematology Am. Soc. Hematol. Educ. Program. 2005:137-142.

2. Small, D. 2006. FLT3 mutations: biology and treatment. Hematology Am. Soc. Hematol. Educ. Program. 2006: $178-184$.

3. Goemans, B.F., et al. 2005. Mutations in KIT and RAS are frequent events in pediatric core-binding factor acute myeloid leukemia. Lenkemia. 19:1536-1542.

4. Streubel, B., et al. 2000. Amplification of the MLL gene on double minutes, a homogeneously staining region, and ring chromosomes in five patients with acute myeloid leukemia or myelodysplastic syndrome. Genes Chromosomes Cancer. 27:380-386.

5. Ferrando, A.A., et al. 2003. Gene expression signatures in MLL-rearranged T-lineage and B-precursor acute leukemias: dominance of HOX dysregulation. Blood. 102:262-268.

6. Garcia-Fernandez, J. 2005. Hox, ParaHox, ProtoHox: facts and guesses. Heredity. 94:145-152.

7. Pineault, N., Helgason, C.D., Lawrence, H.J., and Humphries, R.K. 2002. Differential expression of Hox, Meis1, and $\mathrm{Pbx} 1$ genes in primitive cells throughout murine hematopoietic ontogeny. Exp. Hematol. 30:49-57.

8. Nakamura, T., et al. 1996. Fusion of the nucleoporin gene NUP98 to HOXA9 by the chromosome translocation $\mathrm{t}(7 ; 11)(\mathrm{p} 15 ; \mathrm{p} 15)$ in human myeloid leukaemia. Nat. Genet. 12:154-158.

9. Schoch, C., et al. 2003. AML with $11 \mathrm{q} 23 / \mathrm{MLL}$ abnormalities as defined by the WHO classification: incidence, partner chromosomes, FAB subtype, age distribution, and prognostic impact in an unselected series of 1897 cytogenetically analyzed AML cases. Blood. 102:2395-2402.

10. Camos, M., et al. 2006. Gene expression profiling of acute myeloid leukemia with translocation $\mathrm{t}(8 ; 16)(\mathrm{p} 11 ; \mathrm{p} 13)$ and MYST3-CREBBP rearrangement reveals a distinctive signature with a specific pattern of HOX gene expression. Cancer Res. 66:6947-6954.

11. Kim, D.Y., et al. 2005. Upregulated hoxC4 induces CD14 expression during the differentiation of acute promyelocytic leukemia cells. Leuk. Lymphoma. 46:1061-1066.

12. Scholl, C., et al. 2007. The homeobox gene CDX2 is aberrantly expressed in most cases of acute myeloid leukemia and promotes leukemogenesis. J. Clin. Invest. 117:1037-1048. doi:10.1172/JCI30182.

13. James, R., and Kazenwadel, J. 1991. Homeobox gene expression in the intestinal epithelium of adult mice. J. Biol. Chem. 266:3246-3251.

14. Chase, A., et al. 1999. Fusion of ETV6 to the caudal-related homeobox gene CDX2 in acute myeloid leukemia with the $\mathrm{t}(12 ; 13)(\mathrm{p} 13 ; \mathrm{q} 12)$. Blood. 93:1025-1031.

15. Rawat, V.P., et al. 2004. Ectopic expression of the homeobox gene $\mathrm{Cdx} 2$ is the transforming event in a mouse model of $\mathrm{t}(12 ; 13)(\mathrm{p} 13 ; \mathrm{q} 12)$ acute myeloid leukemia. Proc. Natl. Acad. Sci. U. S. A. 101:817-822.

16. Giampaolo, A., et al. 2002. Expression pattern of HOXB6 homeobox gene in myelomonocytic differentiation and acute myeloid leukemia. Lenkemia. 16:1293-1301.

17. Bansal, D., et al. 2006. Cdx4 dysregulates Hox gene expression and generates acute myeloid leukemia 
alone and in cooperation with Meis1a in a murine model. Proc. Natl. Acad. Sci. U. S. A. 103:16924-16929.

18. Partanen, J., Schwartz, L., and Rossant, J. 1998. Opposite phenotypes of hypomorphic and Y766 phosphorylation site mutations reveal a function for Fgfr1 in anteroposterior patterning of mouse embryos. Genes Dev. 12:2332-2344.

19. Roche, J., et al. 2004. Hox expression in AML identifies a distinct subset of patients with intermediate cytogenetics. Leukemia. 18:1059-1063.

20. Muller-Tidow, C., et al. 2004. High-throughput analysis of genome-wide receptor tyrosine kinase expression in human cancers identifies potential novel drug targets. Clin. Cancer Res. 10:1241-1249.

21. Veraksa, A., Del Campo, M., and McGinnis, W. 2000. Developmental patterning genes and their conserved functions: from model organisms to humans. Mol. Genet. Metab. 60:85-100.

\title{
Hypoglycemia, functional brain failure, and brain death
}

\author{
Philip E. Cryer \\ Division of Endocrinology, Metabolism and Lipid Research, Washington University School of Medicine, St. Louis, Missouri, USA.
}

\begin{abstract}
Hypoglycemia commonly causes brain fuel deprivation, resulting in functional brain failure, which can be corrected by raising plasma glucose concentrations. Rarely, profound hypoglycemia causes brain death that is not the result of fuel deprivation per se. In this issue of the JCI, Suh and colleagues use cell culture and in vivo rodent studies of glucose deprivation and marked hypoglycemia and provide evidence that hypoglycemic brain neuronal death is in fact increased by neuronal NADPH oxidase activation during glucose reperfusion (see the related article beginning on page 910). This finding suggests that, at least in the setting of profound hypoglycemia, therapeutic hyperglycemia should be avoided.
\end{abstract}

Hypoglycemia, including iatrogenic hypoglycemia in people with diabetes, causes brain fuel deprivation that initially triggers a series of physiological and behavioral defenses but if unchecked results in functional brain failure that is typically corrected after the plasma glucose concentration is raised. Rarely, profound, and at least in primates prolonged, hypoglycemia causes brain death.

Given the survival value of maintaining physiological plasma glucose concentrations, it is not surprising that mechanisms that normally very effectively prevent or rapidly correct symptomatic hypoglycemia have evolved (1). As a result, hypoglycemia is a distinctly uncommon clinical event except in people who use drugs that lower the plasma glucose concentration (2). Although there are other drugs, and several relatively uncommon conditions, that cause hypoglycemia (2), in the vast majority of instances the offending drug is an insu-

Nonstandard abbreviations used: T1DM, type 1 diabetes mellitus.

Conflict of interest: The author has served on advisory boards of Novo Nordisk Inc., Takeda Pharmaceuticals North America Inc., MannKind Corp., and Merck and Co. and as a consultant to TolerRx Inc., Amgen Inc., and Marcadia Biotech in recent years.

Citation for this article: J. Clin. Invest. 117:868-870 (2007). doi:10.1172/JCI31669. lin secretagogue or insulin used to treat diabetes mellitus $(2,3)$. As a result of the interplay of relative or absolute therapeutic insulin excess and compromised physiological and behavioral defenses against falling plasma glucose concentrations, hypoglycemia is the limiting factor in the glycemic management of diabetes (3). It causes recurrent morbidity in most people with type 1 diabetes mellitus (T1DM) and in many with advanced T2DM and is sometimes fatal. Furthermore, hypoglycemia, as well as prior exercise and sleep, further compromise glycemic defenses by causing hypoglycemia-associated autonomic failure and thus a vicious cycle of recurrent hypoglycemia. Finally, the barrier of hypoglycemia precludes maintenance of euglycemia over a lifetime of diabetes and thus full realization of the long-term vascular benefits of glycemic control.

\section{Functional brain failure}

Recent interest in alternative brain fuels (including lactate derived from glucose largely within the brain; refs. 4-6) notwithstanding, glucose is an obligate metabolic fuel for the brain under physiological conditions (7). Because the brain cannot synthesize glucose or store substantial amounts as glycogen in astrocytes, the brain requires a virtually continuous supply of glucose from the circulation. Facilitated diffusion of glucose from the blood into the brain is a direct function of the arterial plasma glucose concentration. The rate of blood-to-brain glucose transport exceeds the rate of brain glucose metabolism at normal (or elevated) plasma glucose levels, but it falls and becomes limiting to brain glucose metabolism when arterial glucose concentrations fall to low levels (8). Thus, hypoglycemia causes brain fuel deprivation and, as a result, functional brain failure.

The sequence of responses to falling plasma glucose concentrations (1) is illustrated in Figure 1. Initially, declining plasma glucose levels activate defenses against hypoglycemia. Physiological defenses normally include decrements in pancreatic $\beta$ cell insulin secretion as glucose levels decline within the physiological postabsorptive plasma glucose concentration range (approximately $3.9-6.1 \mathrm{mmol} / \mathrm{l}[70-110 \mathrm{mg} / \mathrm{dl}])$. The glycemic threshold for decreased insulin secretion is approximately $4.5 \mathrm{mmol} / \mathrm{l}(81$ $\mathrm{mg} / \mathrm{dl}$ ). Increments in pancreatic $\beta$ cell glucagon and adrenomedullary epinephrine secretion (among other neuroendocrine responses) normally occur as glucose levels fall just below the physiological range (threshold equal to approximately 3.8 $\mathrm{mmol} / \mathrm{l}[68 \mathrm{mg} / \mathrm{dl}])$. If these defenses fail to abort the hypoglycemic episode, lower glucose levels trigger a more intense sympathoadrenal response that causes neurogenic (or autonomic) symptoms; neuroglycopenic symptoms occur at about the same glucose level (threshold equal to approximately $3.0 \mathrm{mmol} / \mathrm{l}(54 \mathrm{mg} / \mathrm{dl})$. The perception of symptoms, particularly neurogenic symptoms, prompts the behavioral defense, the ingestion of food. If all of these defenses fail, lower glucose levels cause overt functional brain failure that 